\title{
Bioanalysis
}

\section{Novel automated immune turbidimetric assay for routine urinary cystatin-C determinations}

\author{
Balázs Szirmay ${ }^{1}$, Péter Kustán ${ }^{1,2}$, Zoltán Horváth-Szalai ${ }^{1}$, Andrea Ludány ${ }^{1}$, Ágnes Lakatos ${ }^{1}$, \\ Diána Mühl' ${ }^{2}$ István Wittmann ${ }^{3}$, Attila Miseta ${ }^{1}$, Gábor L. Kovács ${ }^{1,4}$ \& Tamás Kőszegi ${ }^{*, 1,4}$ \\ ${ }^{1}$ Department of Laboratory Medicine, University of Pécs Medical School, 7624 Pécs, Ifjúság u. 13, Hungary \\ ${ }^{2}$ Department of Anaesthesiology \& Intensive Therapy, University of Pécs Medical School, 7624 Pécs, Ifjúság u. 13, Hungary \\ ${ }^{3}$ 2nd Department of Internal Medicine \& Nephrology Centre, University of Pécs Medical School, 7624 Pécs, Pacsirta u. 1, Hungary \\ 4János Szentágothai Research Centre, University of Pécs, 7624 Pécs, Ifjúság u. 20, Hungary \\ * Author for correspondence: Tel.: +36 30491 7719; Fax: +36 72536 121; tamas.koszegi@aok.pte.hu
}

\begin{abstract}
Aim: There is no commercially available urinary cystatin-C (u-CYSC) test in the market. Therefore, we optimized and validated an automated immune turbidimetric test for U-CYSC measurements and investigated u-CYSC concentrations in acute and chronic diseases which might lead to renal tubular disorders. Materials \& methods: A particle-enhanced immune turbidimetric assay was adapted and validated on a Cobas 8000/c502 analyzer. Urine samples of different patient groups were also analyzed. Results: Our method showed excellent analytical performance. U-CYSC/U-creatinine (U-CREAT) was higher in sepsisrelated acute kidney injury group ( $p<0.001$ ) compared with controls and to patients with chronic hypertension and Type 2 diabetes. Conclusion: We validated a fast, sensitive, fully automated u-CYSC assay which is ideal for routine use and might be a potential complementary laboratory test to evaluate renal tubular function.
\end{abstract}

First draft submitted: 14 October 2017; Accepted for publication: 22 December 2017; Published online: 16 February 2018

Keywords: acute kidney injury $\bullet$ cystatin- $C \bullet$ tubular damage $\bullet$ turbidimetry $\bullet$ urine $\bullet$ validation

Recently, intense investigation has been focused on finding novel laboratory markers with high diagnostic capability for predicting renal dysfunction, since early diagnosis of renal disorders is of utmost importance. Urinary cystatin-C seems to be a promising protein marker that can provide clinically useful data on tubular dysfunctions [1,2].

It is widely known, that cystatin-C (CYSC) found in plasma is a nonglycosylated inhibitor of cysteine proteases with a molecular mass of $13 \mathrm{kDa}$. It is produced by most nucleated cells at a constant rate and is secreted into the blood [2]. Due to its physical properties CYSC is freely filtrated by the glomeruli; then it is reabsorbed and almost completely catabolized by the proximal tubular cells. Therefore, CYSC is a normal component of urinary proteins at very low concentrations [2-4]. In case of tubular injury the reabsorption and degradation of CYSC might be reduced, leading to increased urinary CYSC (u-CYSC) excretion [5].

In contrast to u-CYSC, serum/plasma CYSC has already been introduced as a reliable marker for estimating GFR with better performance than serum creatinine [6,7]. Serum CYSC seems to be a good marker of glomerular function in acute kidney injury (AKI), while u-CYSC is thought to be an optimal indicator of tubular lesions [8]. However, only few trials have been performed on the clinical utility of u-CYSC. These trials suggested that u-CYSC was a more sensitive predictor of the need of renal replacement therapy in AKI than $\beta_{2}$-microglobulin, and it showed a higher in vitro stability too $[3,5,8]$.

Among patients who underwent cardiac surgery, early and persistent increase of u-CYSC was described correlating with the progression of AKI [9]. Besides acute renal impairment, u-CYSC may provide diagnostic information on diabetic nephropathy (DN) as well. Increased u-CYSC concentrations were found among Type 2 diabetic patients without albuminuria presumably as a result of the tubular damage prior to glomerular manifestation of DN. Consequently, elevated excretion of u-CYSC may predict DN earlier than microalbuminuria [10]. Moreover, it has 
been shown that u-CYSC, referred to urinary creatinine is associated with the severity and progression of chronic kidney disease in patients with obesity and metabolic syndrome [11].

U-CYSC seems to be an appropriate and a highly reliable marker for the early diagnosis of renal tubular dysfunction. However, at present no commercial automated u-CYSC kit is available in the clinical laboratory. Commercial serum CYSC kits cannot be directly applied for u-CYSC measurements because of their inappropriate measuring range. Therefore, in our work we optimized and validated a serum immune turbidimetric test for $\mathrm{u}$-CYSC measurements. The present study also aimed to determine preliminary reference ranges for our assay and to provide u-CYSC data in various patient groups which can be potentially related to acute or chronic renal tubular disorders.

\section{Materials \& methods}

Subjects

Our analyses were done from April 2015 to September 2016. The study was performed according to the Declaration of Helsinki 2008, and was approved by the Regional Ethics Committee of the University of Pécs, Medical School (no. 4327.316-2900/KK15/2011). Every volunteer was fully informed and a written consent was obtained from all of them.

To determine a preliminary reference range of our u-CYSC method, 117 healthy individuals between the age of 10 and 60 years were enrolled. The healthy state was established based on physical examination and on medical records. Exclusion criteria were any kind of acute or chronic diseases and proteinuria. In order to investigate the potential clinical utility of $\mathrm{u}$-CYSC measurements, a group of patients with sepsis-related AKI $(n=33)$, patients with chronic hypertension $(n=43)$ and Type 2 diabetic patients $(n=41)$, were also included. AKI in septic patients was established using the Kidney Disease Improving Global Outcomes guideline [12] supported by serum CYSC concentrations, similarly to others [13-15]. Inclusion criteria for the chronic hypertension and diabetic patient groups were: established clinical diagnosis of their treated disease, the lack of acute inflammatory conditions and other chronic disorders such as autoimmune diseases or tumors.

\section{Sampling \& laboratory testing}

Spot urine samples were obtained from all participants. After centrifugation (1500 g, $10 \mathrm{~min})$ supernatants were collected into aliquots and stored at $-70^{\circ} \mathrm{C}$ until use. Urinary total protein and creatinine (u-CREAT) were determined on a Cobas 8000 analyzer (Roche Diagnostics GmbH, Mannheim, Germany) by standard routine laboratory procedures. U-CYSC data of patients' samples were referred to urinary creatinine and were given as $\mathrm{u}$-CYSC/u-CREAT ratio; however, absolute u-CYSC concentrations were also reported.

\section{Reagents \& instrumentation}

For u-CYSC method adaptation, validation and measurements a particle-enhanced turbidimetric assay was carried out using an open developmental channel of the Cobas 8000/c502 analyzer (Roche Diagnostics GmbH).

The determinations of u-CYSC were performed by using the Cystatin C FS kit (Cat. No. 171589910930, DiaSys Diagnostic Systems GmbH, Holzheim, Germany), which was originally developed for serum CYSC measurements within a range of $0.1-8.0 \mathrm{mg} / \mathrm{l}$. The kit contained polyclonal goat antihuman CYSC antibodies bound to polystyrene particles and a $100 \mathrm{mmol} / \mathrm{l}$ TRIS buffer, pH 7.5.

Dilution series of TruCal Cystatin C calibrator set (Cat. No. 171509910059, DiaSys) were used for calibrations and proper dilutions of TruLab Cystatin C Level 1 and Level 2 (Cat. No. 598709910046, 598809910046, DiaSys) were applied as controls. TruCal Cystatin $C$ was traceable to the IFCC reference material ERM $® D A 471$. For the dilution of the calibrators, controls, patient samples and for blank samples sterile $154 \mathrm{mmol} / 1 \mathrm{NaCl}$ solution was used.

\section{Assay conditions \& validation procedures}

In order to obtain better analytical sensitivity compared with the original serum assay, the test conditions were completely changed. The calibrator/control/sample volume was increased to $12 \mu \mathrm{l}$, the reagent volume was decreased to $50 \mu \mathrm{l}$ and the buffer volume to $170 \mu \mathrm{l}$. The optimal working range for u-CYSC determinations $(0.03-1.0 \mathrm{mg} / \mathrm{l})$ was chosen in the antibody excess zone by testing a wide concentration range of the analyte.

Full calibration was performed by applying six standards: $0.0 ; 0.03 ; 0.125 ; 0.25 ; 0.5 ; 1.0 \mathrm{mg} / \mathrm{l} \mathrm{CYSC}$ solutions by diluting the $1 \mathrm{mg} / \mathrm{l}$ calibrator with saline. The linear regression fitting was applied. For the turbidimetric reaction 
Table 1. Imprecision data of the urinary cystatin-C assay.

\begin{tabular}{|c|c|c|c|c|c|}
\hline \multirow[t]{2}{*}{ Sample } & \multirow[t]{2}{*}{ Target value $\mathrm{mg} / \mathrm{I}$} & \multicolumn{2}{|c|}{ Intra-assay $(n=10)$} & \multicolumn{2}{|c|}{ Interassay $(n=40)$} \\
\hline & & Mean \pm SD mg/l & CV \% & Mean \pm SD mg/I & CV \% \\
\hline $\mathrm{c} 1^{\dagger}$ & 0.728 & $0.759 \pm 0.012$ & 1.52 & $0.744 \pm 0.014$ & 1.94 \\
\hline$c 2^{\dagger}$ & 0.348 & $0.346 \pm 0.006$ & 1.79 & $0.337 \pm 0.006$ & 1.90 \\
\hline $\mathrm{c3}^{\dagger}$ & 0.073 & $0.070 \pm 0.002$ & 3.29 & $0.069 \pm 0.003$ & 4.84 \\
\hline
\end{tabular}

†Samples were diluted from TruLab Cystatin C Level 2 control.

CV: Coefficient of variation; u-CYSC: Urinary cystatin-C.

a two-point end assay type was used at the wavelength of $505 \mathrm{~nm}$ and delta absorbance was calculated between 42-70 measuring points. The assay was performed at $37^{\circ} \mathrm{C}$ with $10 \mathrm{~min}$ of reaction time.

The stability of calibration was checked by summarizing 30 independent calibrations. Intra- and interassay imprecisions, parameters of analytical sensitivity and linearity were assessed based on the Clinical and Laboratory Standards Institute guidelines [16-18]. For determining the limit of blank (LoB) and limit of detection (LoD) five blank and five low-concentration (expected to be very close to the limit of detection) samples (12 replicates/sample) were analyzed. LoQ was determined as the lowest amount of $\mathrm{u}$-CYSC that could be reliably detected with a coefficient of variation $(\mathrm{CV}) \leq 5 \%$ by measuring a dilution series of a urine sample in triplicates in three different runs.

High-dose hook effect was examined in the concentration range of CYSC between 0.0 and $8.0 \mathrm{mg} / \mathrm{l}$. Recovery was examined based on Westgard guidelines by measuring aliquots of five different urine samples spiked with TruLab Cystatin C Level 1 control material (CYSC $=0.844 \mathrm{mg} / \mathrm{l}$ ) or saline and proportional error was calculated [19].

\section{Interference \& stability studies}

Potential interference was tested with calcium, urea, glucose, albumin and hemoglobin. Aliquots of different urine samples spiked with the interferer solution or saline (in 1:9 ratio) were measured in duplicates. Interference was declared when the deviation exceeded the acceptable error (10\%) [19].

To examine analyte stability ten spot urine samples were studied. Specimens were aliquoted and stored without any additives at room temperature and at $4{ }^{\circ} \mathrm{C}$. U-CYSC was determined in duplicates from freshly obtained urine samples and after 2, 4, 6, $24 \mathrm{~h}$ of storage [20]. Additionally, the effects of 3 months' storage at $-20^{\circ} \mathrm{C}$ and freezing-thawing cycles on analyte stability were also assessed by investigating five spot urine samples without any preservatives added. For the latter purpose, aliquots of the samples were frozen at $-70^{\circ} \mathrm{C}$ and subsequently thawed at $37^{\circ} \mathrm{C}$, then three freezing-thawing cycles were performed with a simultaneous u-CYSC determination. Mean percentage deviation was calculated and compared with the acceptable change limit $( \pm 7.1 \%$ in our case).

\section{Statistical analyses}

The distribution of our variables was determined by Shapiro-Wilk analyses. The data showed nonnormal distribution, therefore, Kruskal-Wallis and Mann-Whitney U tests were performed for assessment of the differences between groups. After excluding outliers (only one patient from the adult control group) upper reference limit for $\mathrm{u}$-CYSC and u-CYSC/u-CREAT were determined as 95 percentiles similarly to others [21]. A p-value of $\mathrm{p}<0.05$ was considered as statistically significant. Statistical analyses were carried out by IBM SPSS Statistics for Windows Version 22 (IBM Corporation, NY, USA).

\section{Results \& discussion}

Analytical performance

The calibrated range for u-CYSC measurements was linear between 0.0 and $1.0 \mathrm{mg} / \mathrm{l}$, as shown on Figure 1A. Samples at above $1.0 \mathrm{mg} / \mathrm{l} \mathrm{u}$-CYSC concentration had to be remeasured using an appropriate dilution. Calibration showed good stability with a $\mathrm{CV}<10 \%$ obtained from 30 independent data. Both intra- and interassay imprecision at different concentration levels did not exceed $5 \%$ of CV (Table 1). LoB, LoD and LoQ of our u-CYSC assay were found to be $0.012,0.017$ and $0.037 \mathrm{mg} / \mathrm{l}$, respectively. The quantification limit allowed the accurate determination of samples at a concentration down to $0.037 \mathrm{mg} / \mathrm{l}$. The assay showed good linearity $\left(\mathrm{R}^{2}=0.991\right)$ in the range of $0.04-0.86 \mathrm{mg} / \mathrm{l}$ (Figure 1B). High-dose hook effect was experienced from $3.0 \mathrm{mg} / \mathrm{l} \mathrm{u}$-CYSC concentration; however, the security range expands up to $8.0 \mathrm{mg} / \mathrm{l}$. The recovery study showed the proportional error to be $2.1 \%$ which is within the acceptable error limit. 
(A)

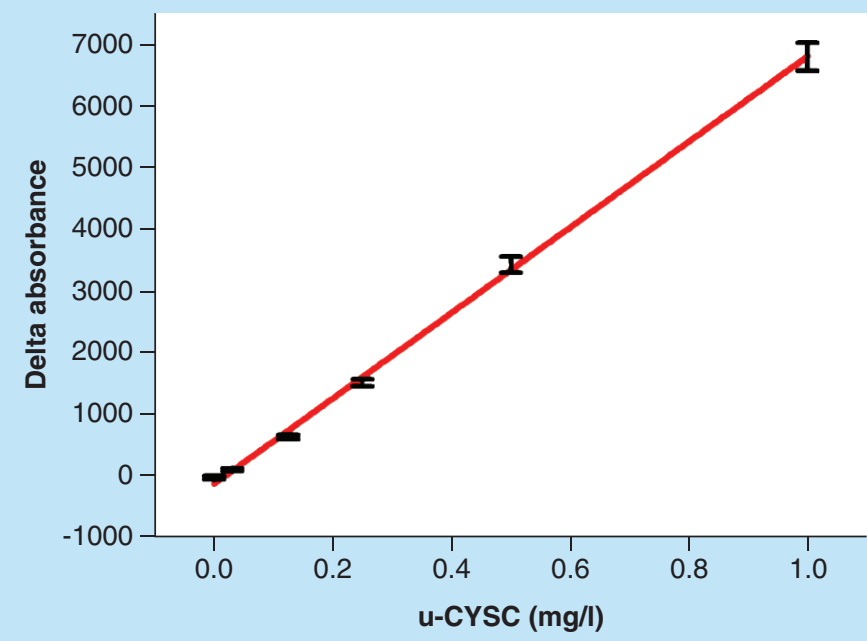

(B)

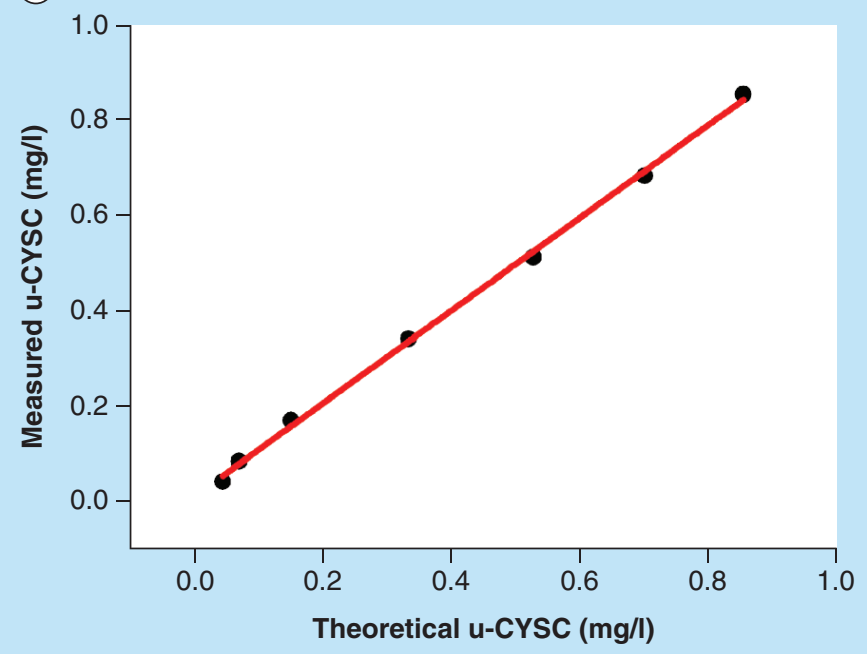

Figure 1. Calibration and linearity graph of urinary cystatin-C. (A) Cumulative graph of the assay in the range of $0.0-1.0 \mathrm{mg} / \mathrm{l}$ with 6 points of calibration ( $n=30$ separate calibrations, mean values of calibrators represented by dots and \pm SDs shown as bars; $R^{2}=0.9989$ ). (B) Linearity was studied by diluting a urine sample. Good linearity was found in the range of $0.86-0.04 \mathrm{mg} / \mathrm{l}$. The equation of the regression line is: $y=1.026 x+0.011, R^{2}=0.991$. Mean values of parallel measurements are represented by dots.

u-CYSC: Urinary cystatin-C.

The assay time of our fully automated new turbidimetric u-CYSC test is only 10 min with an overall imprecision less than $5 \mathrm{CV} \%$ within the whole measuring range. The very low $\mathrm{LoD}(0.017 \mathrm{mg} / \mathrm{l})$ enables u-CYSC determination in almost all healthy individuals. U-CYSC concentrations of samples less than $0.017 \mathrm{mg} / \mathrm{l}$ have no clinical relevance. On the other hand, in severe cases of renal tubular damage but practically for all urine samples above $1.0 \mathrm{mg} / \mathrm{l} \mathrm{of}$ $\mathrm{u}$-CYSC (that is the calibrated range) the test should be repeated after a proper dilution of the urine samples due to the experienced high-dose hook effect. Although u-CYSC is reported to be a reliable marker of tubular dysfunction, up to now only a few automated turbidimetric or nephelometric serum CYSC assays were adapted for urinary measurements $[2,3,5,22,23]$. Our assay properties seem to be similar or even better than those of others $[2,22,23]$. Our LoD is slightly lower than that of the turbidimetric method reported by Makris et al. [23].

\section{Interference \& stability studies}

We found no interference with u-CYSC measurements in spiked urine samples up to $15 \mathrm{mmol} / \mathrm{l}$ calcium, $1350 \mathrm{mmol} / \mathrm{l}$ urea, $280 \mathrm{mmol} / \mathrm{l}$ glucose, $12 \mathrm{~g} / \mathrm{l}$ albumin and $5 \mathrm{~g} / \mathrm{l}$ hemoglobin, respectively. In accordance with our results, former studies did not report considerable interference with albumin and hemoglobin $[2,3]$. Other endogenous substances that are present in the urine such as calcium, urea and glucose also did not have impact on $\mathrm{u}-\mathrm{CYSC}$ analysis.

We observed that u-CYSC was stable for $24 \mathrm{~h}$ stored at $4{ }^{\circ} \mathrm{C}$ and for $6 \mathrm{~h}$ stored at room temperature $(<5 \%$ average decrease). However, two samples showed up to $15 \%$ loss of CYSC under identical conditions. After a 3 -month storage at $-20^{\circ} \mathrm{C}$ and after the third freezing-thawing cycle in case of two samples the reduction of $\mathrm{u}$-CYSC concentration exceeded the acceptable change limit while the other samples remained stable.

The favorable analytical performance and the short reaction time make our u-CYSC assay to be proper for routine clinical use which can help rapid decision making in the clinical practice. However, as a limitation we should emphasize that our stability studies revealed CYSC being unstable in some urine samples. The instability seems to be independent of storage temperature as it was previously suggested by others too [2]. Our data support the recommendation that proteinase inhibitor preservatives should be added to freshly voided urine samples $[4,5]$.

\section{Preliminary reference ranges, patients' data}

In the group of healthy individuals, we observed no significant differences in u-CYSC excretion between genders. Healthy adolescents (age group 10-18 years) showed slightly but significantly elevated u-CYSC/u-CREAT values 
(A)

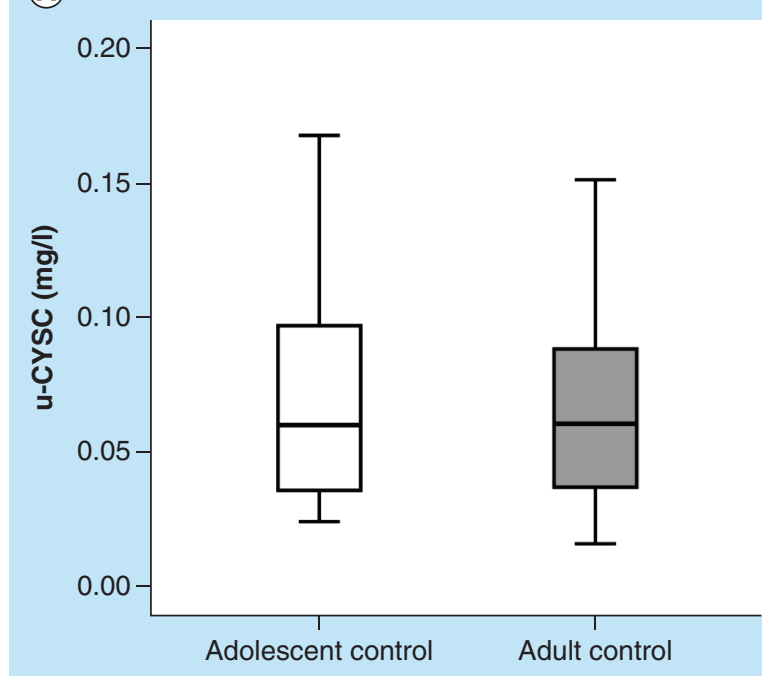

(B)

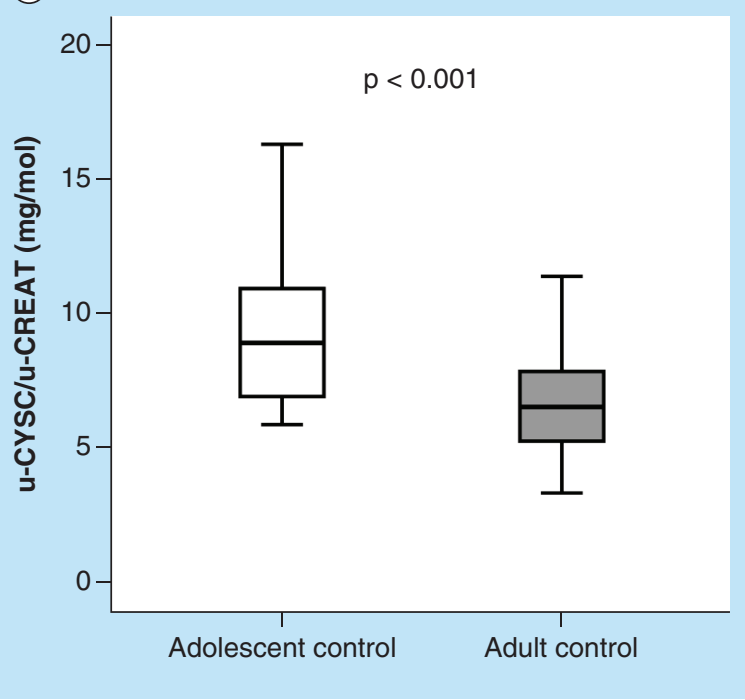

Figure 2. Urinary cystatin-C and urinary cystatin-C/urinary creatinine data of healthy individuals. Mann-Whitney $U$ test was used to compare the variables of groups. (A) Comparison of u-CYSC concentrations between adolescent and adult control groups. (B) Comparison of u-CYSC/u-CREAT values between adolescent and adult control groups. u-CYSC: Urinary cystatin-C; u-CYSC/u-CREAT: Urinary cystatin-C/urinary creatinine.

Table 2. Demographic and laboratory data of the studied patient groups.

\begin{tabular}{|c|c|c|c|c|}
\hline Parameters & Adult control group & $\begin{array}{l}\text { Sepsis-related } \\
\text { AKI patients }\end{array}$ & $\begin{array}{l}\text { Patients with chronic } \\
\text { hypertension }\end{array}$ & Patients with Type 2 DM \\
\hline Number (n) & 84 & 33 & 43 & 41 \\
\hline Females n (ratio) & $44(0.52)$ & $16(0.48)$ & $20(0.47)$ & $14(0.34)$ \\
\hline Age (years) & $40(21-60)$ & $70(25-82)$ & $62(26-79)$ & $66(45-81)$ \\
\hline $\mathrm{u}-\mathrm{CYSC}(\mathrm{mg} / \mathrm{l})$ & $0.06(0.04-0.09)$ & $\begin{array}{l}0.91^{\dagger} \\
(0.28-3.25)\end{array}$ & $\begin{array}{l}0.05 \\
(0.03-0.09)\end{array}$ & $\begin{array}{l}0.06 \\
(0.04-0.08)\end{array}$ \\
\hline $\begin{array}{l}\mathrm{u}-\mathrm{CYSC} / \mathrm{u}-\mathrm{CREAT} \\
(\mathrm{mg} / \mathrm{mol})\end{array}$ & $\begin{array}{l}6.5 \\
(5.3-7.8)\end{array}$ & $\begin{array}{l}309.6^{\dagger} \\
(123.1-1010.8)\end{array}$ & $\begin{array}{l}6.9 \\
(5.4-9.1)\end{array}$ & $\begin{array}{l}6.2 \\
(5.0-8.2)\end{array}$ \\
\hline
\end{tabular}

${ }^{\dagger} p<0.001$ compared with healthy adult controls. Median (25-75\% percentiles) are presented except for age: median (min-max). Mann-Whitney $U$ test was used to compare each patient groups to control group.

AKI: Acute kidney injury; DM: Diabetes mellitus; u-CYSC: Urinary cystatin-C; u-CYSC/u-CREAT: Urinary cystatin-C/urinary creatinine.

$(8.9 \mathrm{mg} / \mathrm{mol})$ compared with adults $(6.5 \mathrm{mg} / \mathrm{mol}, \mathrm{p}<0.001)$ but their $\mathrm{u}-\mathrm{CYSC}$ concentrations (expressed as $\mathrm{mg} / \mathrm{l}$ ) appeared to be similar to adults (Figure 2). U-CYSC concentrations of a few healthy controls were found to be below the LoQ thus only upper reference limits ( $<95$ percentiles) were defined: $<0.14 \mathrm{mg} / \mathrm{l}$ for u-CYSC independently of age, $<19.7 \mathrm{mg} / \mathrm{mol}$ for u-CYSC/u-CREAT for the adolescents and $<12.4 \mathrm{mg} / \mathrm{mol}$ for adults.

We found significantly $(\mathrm{p}<0.001)$ elevated $\mathrm{u}-\mathrm{CYSC}$ concentrations and $\mathrm{u}-\mathrm{CYSC} / \mathrm{u}$-CREAT ratios in patients with sepsis-related AKI compared with adult controls. In contrast to these data, values of patients with chronic hypertension and Type 2 diabetes did not differ at all from those of the healthy group (Table 2).

Urinary CYSC does not show circadian rhythm, therefore spontaneous urine samples can be used for both establishing reference ranges and for clinical application as well [24]. The observed u-CYSC values among healthy people are in agreement with previously reported data [25]. We determined the upper reference limit only as 95th percentiles. Complete reference interval could not be calculated since several urine samples showed lower CYSC concentrations than the LoQ and the very low values have no clinical relevance. Similarly to us, Conti et al. determined the upper reference limit of u-CYSC at $0.18 \mathrm{mg} / \mathrm{l}$ by nephelometry; furthermore, Noraddin and coworkers calculated that as $0.16 \mathrm{mg} / \mathrm{l}$ by turbidimetry [5,22].

Since spot urine samples were analyzed, it was reasonable to express results also as u-CYSC/u-CREAT ratio in order to exclude the effect of different hydration states (as it is reported by others) [4,8,26-28]. However, it is 
known that in case of tubular damage the excretion of creatinine is also affected which may influence the urinary protein/u-CREAT values [29]. Therefore, giving both the absolute concentrations of $\mathrm{u}-\mathrm{CYSC}$ and the $\mathrm{u}-\mathrm{CYSC} / \mathrm{u}-$ CREAT values is suggested. Even if both data are given, it is still an open problem if absolute u-CYSC values or u-CYSC/u-CREAT or both are preferred. In order to give a proper answer, the study should be extended in the future to include more patient groups and to largely increase the number of patients in each group. Our $\mathrm{u}$-CYSC/u-CREAT data of healthy adults were also similar to that reported by Grubb [4]. Although our study population was relatively small, we found significant differences in u-CYSC/u-CREAT values between adolescents and adults which confirms a recent observation [30]. This finding might be due to the age dependency of urinary creatinine excretion associated with the growth of muscle mass during adolescence [31].

We detected significant elevation of $\mathrm{u}-\mathrm{CYSC}$ and $\mathrm{u}$-CYSC/u-CREAT ratio in AKI patients compared with the control group, indicating the renal tubular damage in sepsis-related AKI. However, the observed increase of $\mathrm{u}-\mathrm{CYSC}$ was not as extreme as that was described in a former paper [1]. Although chronic hypertension is a risk factor for nephropathy, our results suggest that u-CYSC seems not to be affected by chronic hypertension. In the diabetic patient group u-CYSC, data suggest that tubular damage has not been developed yet. Tubular damage might precede the appearance of microalbuminuria only in a few cases of diabetes where increased u-CYSC may be useful for the recognition of these patients [26]. Moreover, in certain cases with proteinuria it should be taken into consideration, that the competition between albumin and low molecular weight proteins for receptor-mediated reabsorption could elevate the urinary levels of CYSC as well [32].

The current study has some limitations. Our major focus was on the analytical validation of the u-CYSC assay, therefore it cannot be considered as a clinical study. However, the presented data on healthy individuals and on patient groups support our suggestion that u-CYSC determination is recommended for clinical utilization. Unfortunately, calibrators, control materials and a reference method are available only for serum samples and not for u-CYSC measurements. The described preliminary reference ranges were established by investigation of a relatively small healthy population and our adolescent group was remarkably smaller than the adult group. Another limitation of our work is that number of patients in the different disease groups was smaller than that in the control group. Also, during our stability studies samples with protease inhibitor were not analyzed.

\section{Conclusion}

The present study describes a novel validated particle-enhanced immune turbidimetric assay for u-CYSC determination which can easily be adapted to other commercial fully automated analyzers. It is sensitive, precise, rapid and offers a short turnaround time, therefore it seems to be ideal for routine clinical utilization. U-CYSC reference range seems to be age-dependent when data are referred to urinary creatinine. For discrimination between glomerular and tubular renal injury, both serum and u-CYSC measurement is suggested. The analytical performance of our method enables to detect subtle changes in u-CYSC concentrations with a potential positive predictive value for timely diagnosis of renal tubular damage. In septic patients with AKI, our data further supports the findings of the relatively few studies that $\mathrm{u}$-CYSC might be a reliable marker of renal tubular injury.

\section{Future perspective}

Urinary cystatin- $C$ determinations are rare, even nowadays and there is no commercially available automated method for its quantification as a diagnostic tool. While serum CYSC is widely used for the estimation of glomerular function in AKI patients, serum CYSC is not suitable for predicting severe tubular damage. Therefore, the major goal of determining u-CYSC is the fact that it reflects and predicts tubular type acute kidney failure. Our automated method with its short assay time seems to be an ideal lab test that might be introduced into the routine laboratory palette. The analytical sensitivity of the test enables u-CYSC determination in practically all healthy individuals. On the other hand, in critically ill patients (e.g., in sepsis) u-CYSC values increase dramatically when development of tubular damage and occurrence of AKI is expected. We think, that in the future, diagnostic kits for $\mathrm{u}$-CYSC determination will be produced. In order to add u-CYSC to the diagnostic arsenal, proper quality control is required which is also to be developed. Most probably, stabilizers should be added to the urine samples in order to prevent potential preanalytical degradation of u-CYSC. Our method is easy to adapt to common laboratory automats and u-CYSC quantification is expected to help rapid decision-making regarding critically ill patients. 
- Urinary cystatin-C (u-CYSC) is considered to be a promising protein marker that can provide clinically useful data on renal tubular dysfunctions. Especially in acute kidney injury of tubular origin u-CYSC levels might increase dramatically. However, there is no commercial kit available for u-CYSC determination.

- In our work, a novel automated particle enhanced immune turbidimetric assay for measurement of u-CYSC was developed and adapted to a commercial laboratory analyzer. Our assay was fully validated and preliminary reference ranges were also established. The test is rapid $(10 \mathrm{~min}$ ) with a limit of quantification of $0.037 \mathrm{mg} / \mathrm{l}$ $\mathrm{u}$-CYSC. The high analytical sensitivity makes the test being suitable for measurement of u-CYSC levels practically in all healthy individuals.

- When reporting u-CYSC data, it is suggested to give both u-CYSC concentrations and u-CYSC/u-CREAT ratios as well.

- In our studied patient groups (Type 2 diabetes, hypertension, control and septic patients) a dramatic increase was found in septic patients with acute kidney injury. The largely increased u-CYSC values indicated renal tubular damage.

- The u-CYSC assay is strongly suggested to be part of the laboratory palette when dealing with critically ill patients. Development of a commercially available u-CYSC diagnostic kit with effective quality control is of utmost importance.

\section{Acknowledgements}

The present scientific contribution is dedicated to the 650th anniversary of the foundation of the University of Pécs, Hungary.

Financial \& competing interests disclosure

The work was financially supported by GINOP-2.3.2-15-2016-00021'The use of chip-technology in increasing the effectiveness of human in vitro fertilization'; NKFI-EPR K/115394/2015 'Early biochemical indicators of embryo viability'; KA-2016-04 (University of Pécs, Medical School); and 'ÚNKP-17-3-III New National Excellence Program of the Ministry of Human Capacities' grants. The authors express their special thanks to DiaSys Diagnostic Systems GmbH for providing the CYSC reagent and to Roche Magyarország Kft, for the invaluable technical help. The authors have no other relevant affiliations or financial involvement with any organization or entity with a financial interest in or financial conflict with the subject matter or materials discussed in the manuscript apart from those disclosed.

No writing assistance was utilized in the production of this manuscript.

\section{Ethical conduct of research}

The authors state that they have obtained appropriate institutional review board approval or have followed the principles outlined in the Declaration of Helsinki for all human or animal experimental investigations. In addition, for investigations involving human subjects, informed consent has been obtained from the participants involved.

\section{Open access}

This work is licensed under the Attribution-NonCommercial-NoDerivatives 4.0 Unported License. To view a copy of this license, visit http://creativecommons.org/licenses/by-nc-nd/4.0/

\section{References}

Papers of special note have been highlighted as: $\bullet$ of interest; $\bullet \bullet$ of considerable interest

1. Nejat M, Pickering JW, Walker RJ et al. Urinary cystatin C is diagnostic of acute kidney injury and sepsis, and predicts mortality in the intensive care unit. Crit. Care 14(3), R85 (2010).

-. The potential clinical benefit of measuring urinary cystatin- $\mathrm{C}$ in critically ill patients.

2. Sohrabian A, Noraddin FH, Flodin M, Fredricsson A, Larsson A. Particle enhanced turbidimetric immunoassay for the determination of urine cystatin C on Cobas c501. Clin. Biochem. 45(4-5), 339-344 (2012).

3. Herget-Rosenthal S, Feldkamp T, Volbracht L, Kribben A. Measurement of urinary cystatin C by particle-enhanced nephelometric immunoassay: precision, interferences, stability and reference range. Ann. Clin. Biochem. 41(Pt 2), 111-118 (2004).

4. Grubb AO. Cystatin C - properties and use as diagnostic marker. Adv. Clin. Chem. 35, 63-99 (2000).

- Summarizes the basic properties and initial investigations on cystatin-C.

5. Conti M, Moutereau S, Zater M et al. Urinary cystatin C as a specific marker of tubular dysfunction. Clin. Chem. Lab. Med. 44(3), 288-291 (2006). 
6. Bargnoux AS, Pieroni L, Cristol JP et al. Multicenter evaluation of cystatin C measurement after assay standardization. Clin. Chem. 63(4), 833-841 (2017).

7. Grubb A, Lindstrom V, Jonsson $\mathrm{M}$ et al. Reduction in glomerular pore size is not restricted to pregnant women. Evidence for a new syndrome: 'Shrunken pore syndrome'. Scand. J. Clin. Lab. Invest. 75(4), 333-340 (2015).

8. Lisowska-Myjak B. Serum and urinary biomarkers of acute kidney injury. Blood Purif. 29(4), 357-365 (2010).

-. The potential clinical benefit of measuring urinary cystatin- $C$ in critically ill patients.

9. Koyner JL, Bennett MR, Worcester EM et al. Urinary cystatin C as an early biomarker of acute kidney injury following adult cardiothoracic surgery. Kidney Int. 74(8), 1059-1069 (2008).

-• The potential clinical benefit of measuring urinary cystatin- $\mathrm{C}$ in critically ill patients.

10. Jeon YK, Kim MR, Huh JE et al. Cystatin C as an early biomarker of nephropathy in patients with Type 2 diabetes. J. Korean Med. Sci. 26(2), 258-263 (2011).

- Evaluates the usefulness of urinary cystatin- $\mathrm{C}$ as a biomarker in the recognition of diabetic nephropathy.

11. Satoh-Asahara N, Suganami T, Majima $\mathrm{T}$ et al. Urinary cystatin $\mathrm{C}$ as a potential risk marker for cardiovascular disease and chronic kidney disease in patients with obesity and metabolic syndrome. Clin. J. Am. Soc. Nephrol. 6(2), 265-273 (2011).

12. Kidney Disease: Improving Global Outcomes (KDIGO) Acute Kidney Injury Work Group. KDIGO Clinical Practice Guideline for acute kidney injury. Kidney Int. Suppl. 2, 1-138 (2012).

13. Zappitelli M, Greenberg JH, Coca SG et al. Association of definition of acute kidney injury by cystatin C rise with biomarkers and clinical outcomes in children undergoing cardiac surgery. JAMA Pediatr. 169(6), 583-591 (2015).

14. Zhou J, Liu Y, Tang Y et al. A comparison of RIFLE, AKIN, KDIGO, and Cys-C criteria for the definition of acute kidney injury in critically ill patients. Int. Urol. Nephrol. 48(1), 125-132 (2016).

15. Kustan P, Szirmay B, Horvath-Szalai Z et al. Urinary orosomucoid: a novel, early biomarker of sepsis with promising diagnostic performance. Clin. Chem. Lab. Med. 55(2), 299-307 (2017).

16. Clinical and Laboratory Standards Institute. Preliminary Evaluation of Quantitative Clinical Laboratory Measurement Procedures; Approved Guideline (3rd Edition). CLSI document EP10-A3. Wayne, PA, USA (2006).

17. Clinical and Laboratory Standards Institute. Evaluation of Quantitative Measurement Procedures: A Statistical Approach; Approved Guideline. CLSI document EP06-A. Wayne, PA, USA, 2003.

18. Clinical and Laboratory Standards Institute. Evaluation of Detection Capability for Clinical Laboratory Measurement Procedures; Approved Guideline (2nd Edition). CLSI document EP17-A2. Wayne, PA, USA, 2012.

19. Westgard Basic Method Validation. www.westgard.com/lesson27.htm

20. Oddoze C, Lombard E, Portugal H. Stability study of 81 analytes in human whole blood, in serum and in plasma. Clin. Biochem. 45(6), 464-469 (2012).

21. Tencer J, Thysell H, Grubb A. Analysis of proteinuria: reference limits for urine excretion of albumin, protein HC, immunoglobulin G, kappa- and lambda-immunoreactivity, orosomucoid and alpha 1-antitrypsin. Scand. J. Clin. Lab. Invest. 56(8), 691-700 (1996).

22. Noraddin FH, Flodin M, Fredricsson A, Sohrabian A, Larsson A. Measurement of urinary cystatin C with a particle-enhanced turbidimetric immunoassay on Architect ci8200. J. Clin. Lab. Anal. 26(5), 358-364 (2012).

23. Makris K, Nikolaki E, Nanopoulos K, Pirgakis KM, Maltezos CK. Measurement of cystatin C in human urine by particle-enhanced turbidimetric immunoassay on an automated biochemistry analyzer. Clin. Biochem. 46(12), 1128-1130 (2013).

24. Conti M, Zater M, Lallali $\mathrm{K}$ et al. Absence of circadian variations in urine cystatin $\mathrm{C}$ allows its use on urinary samples. Clin. Chem. 51(1), 272-273 (2005).

25. Mijušković Z, Maksić $Đ$, Hrvačević R et al. Urinary cystatin $\mathrm{C}$ as a marker of tubular dysfunction. J. Med. Biochem. 26(2), (2007).

26. Kim SS, Song SH, Kim IJ et al. Urinary cystatin C and tubular proteinuria predict progression of diabetic nephropathy. Diabetes Care 36(3), 656-661 (2013).

27. Uchida K, Gotoh A. Measurement of cystatin-C and creatinine in urine. Clin. Chim. Acta 323(1-2), 121-128 (2002).

28. Helmersson-Karlqvist J, Arnlov J, Carlsson AC, Harma J, Larsson A. Increased urinary cystatin C indicated higher risk of cardiovascular death in a community cohort. Atherosclerosis 234(1), 108-113 (2014).

29. Makris K, Stefani D, Makri E et al. Evaluation of a particle enhanced turbidimetric assay for the measurement of neutrophil gelatinase-associated lipocalin in plasma and urine on Architect-8000: analytical performance and establishment of reference values. Clin. Biocehem. 48(18), 1291-1297 (2015).

30. Pennemans V, Rigo JM, Faes C, Reynders C, Penders J, Swennen Q. Establishment of reference values for novel urinary biomarkers for renal damage in the healthy population: are age and gender an issue? Clin. Chem. Lab. Med. 51(9), 1795-1802 (2013).

31. Remer T, Neubert A, Maser-Gluth C. Anthropometry-based reference values for 24-h urinary creatinine excretion during growth and their use in endocrine and nutritional research. Am. J. Clin. Nutr. 75(3), 561-569 (2002).

32. Nejat M, Hill JV, Pickering JW, Edelstein CL, Devarajan P, Endre ZH. Albuminuria increases cystatin C excretion: implications for urinary biomarkers. Nephrol. Dial. Transplant. 27(Suppl. 3), iii96-iii103 (2012). 